\title{
SOME NOTES ON VOTING SCHEMES AND THE WILL OF THE MAJORITY*
}

\author{
Bernard Grofman \\ University of Chicago
}

This paper is a study in the theory of committees and elections. By a committee we will mean any group of people who arrive at a decision by means of voting. By a voting scheme ${ }^{1}$ we will mean any method by which individual voting decisions are aggregated into committee decisions. Given various voting schemes we shall examine three techniques by which members may seek to manipulate committee decisions to their advantage: a) additions or deletions to the alternatives to be considered b) deliberate distortions of one's own voting preferences c) manipulation of the order in which alternatives are voted upon, and shall prove some theorems about rational voting behavior when preferences are unidimensionally scalable.

In order to simplify the task before us we shall consider only voting schemes which select one individual or proposal from among several mutually exclusive alternatives 2 , shall restrict ourselves to schemes which constitute majority games 3 , and shall consider only ordinal rankings among preferences. We shall assume: (1) that committee members possess fixed and immutable preference schedules. (2) that individual preferences are strongly ordered. ${ }^{4}$ (3) that committee members possess complete information as to the preference schedules of all other committee members. ${ }^{5}$ (4) that all balloting is open (no secret ballots) and that all committee members possess perfect information. ${ }^{6}(5)$ that each election is entire unto itself, i.e., independent of the outcome of any other election. ${ }^{7}(6)$ that the number of committee members is odd, and (7) that all committee members rationally seek to maximize utility. 8

Before we can proceed to the main body of our exposition we must introduce a number of definitions.

Definition: A voting scheme shall be said to satisfy the criterion of independent of irrelevant alternatives (CIIA) if for any set of alternatives and for any set of committee member's preference schedules if when alternatives are deleted, none of which was selected by the committee, then the alternative selected by the committee remains that which would have been selected had the alternatives not been deleted.

* Based upon the author's Master's Thesis at the University of Chicago done under the supervision of Professors Duncan MacRae and Morton Kaplan. I am indebted to Professor Duncan Black for a number of helpful suggestions including the detection of errors in the initial statement of two of my proofs. Errors remaining are, of course, the responsibility solely of the author. 


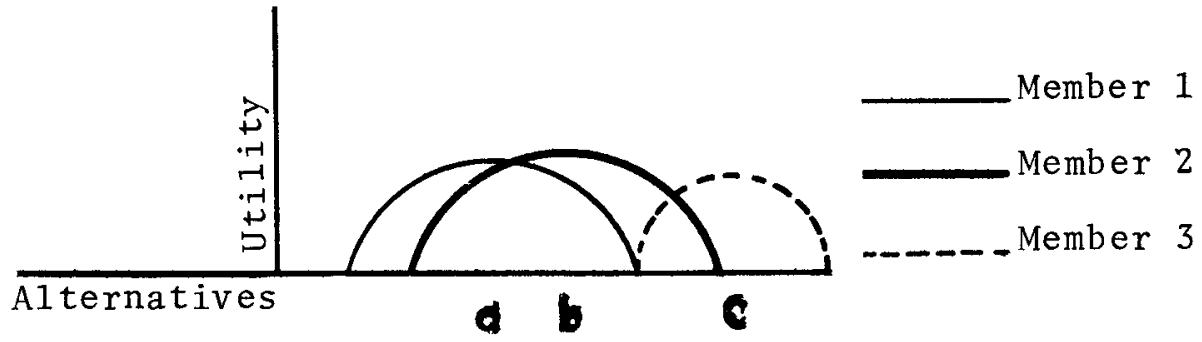

Figure 1 
Definition: that alternative, if any, which with committee members not deviating from their true preferences, is preferred by a majority in paired comparison to each and every other alternative, shall be designated the Condorcet choice (c). 9

Definition: A voting scheme shall be said to satisfy the Condorcet criterion if it always selects the Condorcet choice, whenever such a choice exists.

Definition: A set of preference schedules shall be said to be single-peaked if there exists an ordering of the alternatives such that with respect to this ordering the preference schedules of all committee members could be graphed as single peaked curves. 10

Definition: A single peaked curve is one which changes its sign of slope at most once, from up to down.

We have now introduced definitions of two criteria by which the fairness of voting schemes might be judged. The Condorcet choice and the criterion of independence of irrelevance of dependent alternatives (CIIA) may be related by the following theorems which also shed light on Arrow's Impossibility Theorem.

Theorem 1: When members adhere to their strict schedule of preferences, for any set of preference schedules such that a definite choice will be arrived at, a voting scheme which always selects the Condorcet choice whenever such a choice exists (i.e., always satisfies the Condorcet criterion) will always satisfy the criterion of independence of irrelevant alternatives (CIIA).

Proof: Given the existence of a Condorcet choice (c) any voting scheme which always satisfies the Condorcet criterion also always satisfies the criterion of independence of irrelevant alternatives (CIIA) Delete any irrelevant alternatives. This will not affect the determination of the Condorcet choice and thus CIIA is satisfied. q.e.d.

Theorem 2: When members adhere to their strict schedule of preferences, for any set of preference schedules such that a definite outcome will be arrived at, a voting scheme which also has the property in the two alternative case of being equivalent to the method of simple majority decision always selects the Condorcet choice if it always satisfies CIIA when members adhere to their strict schedules of preferences.

Proof: Considering any voting scheme with this property which also satisfies CIIA. Call the alternative selected by the scheme $j s$ : label the remaining alternatives $a_{1} a_{2} \ldots a_{n-1}$. Consider the contest between $s$ and aj. Either $s$ is selected or not. If $\mathbf{s}$ is not selected then CIIA is violaced, thus $s$ must receive a majority in 
paried contests against each of the other alternatives, $i$. e. $s$ is a Condorcet choice. q.e.d.

Corollary: For any set of preference schedules a voting scheme having the property in the two alternative case of being equivalent to the method of simple majority decision and which also satisfied CIIA will select the Condorcet choice.

In this paper we shall consider three voting schemes:

The first scheme we shall take up in "lowest candidate out runoffs" (LCOR). This scheme is undoubtedly the most common in elections among small groups. The first ballot is held among all $\mathrm{n}$ candidates, each of $\mathrm{k}$ committee members having one vote. If a candidate receives a majority of the votes cast on that ballot he is elected: if not, the candidate receiving the fewest votes is eliminated. And so on, until either a majority has been obtained for some candidate on some ballot, or the balloting is deadlocked because of ties, or the balloting is reduced to that between two candidates one of whom must then receive a majority. 12

LCOR, does not satisfy the Condorcet criterion. Consider a committee whose members have schedule of preferences $x y z, x y z, z y x, z v x$, Given strict adherence to preference schedules $z$ will be chosen, despite the fact that $y$ is the Condorcet choice. As we would expect LCOR also fails to satisfy the criterion of independence of irrelevant alternatives. Consider the introduction of an additional alternative $p$ to the preference schedules above, making them: pxyz, pxyz, zxyp, yzxp, yzxp. LCOR now selects (again assuming strict adherence to schedules of preferences) y rather than $x$. LCOR tends to "favor extremists at the expense of moderates", 13 something of which few of those who make blithe use of this procedure are aware. The chief advantage of LCOR and the undobted reasons for its popularity are that it requires at most $n-1$ ballots (and usually less) and is remarkably simple to administer.

The next voting scheme we shall consider is "standard amendment procedure," (SAP), so named because it is just that. ${ }^{14}$ In some determined order, usually the opposite order in which they are moved, alternatives are paired against one another. When an alternative fails to receive a majority it is eliminated. New alternatives are introduced one by one and paired off against the winner of the preceding ballot until only one alternative remains. This alternative is then voted upon separately (i.e., is paired against the status quo.) If it receives a majority it is approved. If it fails to receive a majority, even though it has directly or indirectly defeated all other alternatives, it fails to pass and the status quo remains in effect.

Black 15 has shown that when committee members' preference schedules are single peaked for all alternatives including the status quo then this procedure (and several other procedures he discusses) will lead to the Concorcet choice, provided that members adhere strictly to their preference schedules. When schedules are 
single-peaked the standard amendment procedure satisfies the criterion of independence of irrelevant alternatives. Even without the single-peakedness restriction a Condorcet choice, if one exists, will be victorious under SAP provided that members do not deviate from their preference schedules; but in the absence of a Condorcet choice SAP does not, in general, satisfy the criterion of independence of irrelevant alternatives. I leave the reader to devise his own examples.

The final voting scheme we shall take up is that of filling in the blanks (FIB), 16 a little known and little used method of amendment in which alternatives are ranked among some (presumably unidimensionally scalable) continum and are then posed in order, one at a time until one receives a majority - with the motion as amended then coming up for a vote. To give an example: suppose a committee is intending to purchase and renovate a building for some as yet unspecified amount and the alternatives proposed are $\$ 30,000, \$ 20,000$, and $\$ 25,000$. Voting by FIB would involve voting first whether $\$ 30,000$ was to be spent. If a majority voted yes thescommittee would vote on the resolution as amended, thus the vote would be whether or not to allocate $\$ 25,000$. If that did not carry, then the vote would be on the final alternative, $\$ 20,000$, which presumably would receive a majority. Then the committee would vote on the resolution as amended.

Theorem 3: For any set of preference schedules such that a definite choice will be arrived at when members adhere to their strict schedule of preferences, filling in the blanks selects the Condorcet choice (c) when preference schedules for all alternatives including the status quo $(q)$ are single-peaked.17

Proof: Denote by s the alternative selected by the above procedure. (Note: $s$ may be identical with q.) When preferences are single-peaked then all those who vote for s prefer it to any alternative yet to be considered; thus s will obtain a majority paired against any alternative not yet considered. (For clarification see the diagram below based on the three-alternative case). Similarly, all committee members whose first choice is an alternative not yet voted upon (i.e., who are not part of the majority which voted for s) prefer $s$ to any alternatives previously voted upon. But we know that less than a majority was obtained for any alternative considered before $s$ since otherwise $s$ would never have been considered. But then those whose first choice is either some alternative not yet considered or s itself must comprise a majority and this majority will be obtained for $s$ in a paired comparison against any alternative already posed. Thus $s$ is a majority choice when paired against any other alternative and is therefore, by definition, the Condorcet choice.

Collary: Given adherence to their strict schedule of references by all committee members, filling in the blanks satisfies the criterion of independence of irrelevant alternatives. 


\section{a) ADDITIONS OR DELETIONS OF ALTERNATIVES TO BE CONSIDERED}

LCOR and SAP are not independent of irrelevant alternatives, at least as long as members adhere to their strict schedule of preferences. FBI, on the other hand, (assuming single-peakedness) is. We shall briefly consider examples of manipulation by a strategic deletion or addition of alternatives in each of the former.

Consider a five-member committee voting by LCOR with preferences pxyz, pxyz, zxyp, yzxp, yzxp. LCOR selects, assuming strict adherence to preference schedules, $y$. If the (irrelevant alternative) $p$ is deleted then $x$ will be selected. $P$ drew strength away from $x$ since for four of the five committee members the preferences for $x$ and $p$ were virtually identical. In LCOR outcomes may be manipulated by the introduction of (irrelevant) alternatives which strongly appeal to the same voters as the widely supported front runner (s) whom one wishes to defeat. If this strategy is successful moderate candidates may never make it to the later stages of balloting in which their widespread acceptability when first and second choices were no longer in the running would give them a chance of victory.

We shall now consider a three member committee voting by SAP with preferences pxyz, zpxy, and yxzp. If the alternatives are voted upon in the order first $p$ and $x$, then $y$, then $z$; then $z$ will be selected. If $p$ is deleted from the set of alternatives being considered, with the order of voting among the remaining alternatives remaining unchanged, then $\mathrm{x}$ will be selected. (In both cases, of course, we are assuming strict adherence to preference schedules.) A more important possibility for manipulation lies in the order in which alternatives are voted upon. That is a subject we shall touch upon below.

\section{b) DELIBERATE DISTORTIONS OF VOTING PREFERENCES}

When confronted with the possibility of committee members concealing their true preferences in order to advance their interests as a defect in his voting scheme Bora replied, "My scheme is only intended for honest men." 18 Black, although he recognizes that there are situations in which deviations from his schedule of preferences would be in a committee member's interest, does not consider this a significant phenomenon. With one exception, all the theorems proved by Black assume that committee members adhere to their strict schedule of preferences. Borda and Black are not alone in their unwillingness to consider deviations from preference schedules. 19

To demonstrate but one important case in which it was rational for a large number of voters to deviate from their preference schedules, consider the 1948 U.S. Presidential election. As Downs has noted, "Some voters who preferred the Progressive candidate to all others nevertheless voted for the Democratic candidate. They did so because they felt their favorite candidate had no chance at all, and the more people voted for him, the fewer would vote Democratic. If the Democratic vote fell low enough, then the Republicans - the least desirable group from the Progressive point of view - would win. Thus a vote for their favorite candidate 
ironically increased the probability that the one they favored least would win."20 The argument as to why single-member constituencies foster a two-party system. employs similar reasoning. In an election by plurality "extremists" must stress ideological purity if they are to retain voters in the face of what seems a lost cause, while "moderates" must stress the inutility of "wasting" one's vote. Ceteris paribus, the closer the election is expected to be, the fewer the vote's actually given to extremist candidates and the greater the deviations from strict preference schedules.

Let us now consider committees voting by LCOR. Consider a five-member committee with preferences xzyp, yzxp, yzxp, pxzy, pzxy. If members adhere to their preference schedules $y$ will be selected. Yet a majority of members prefer either $\mathbf{x}$ or $\mathrm{z}$ to $\mathrm{y}$, and thus some have an incentive to deviate from their preference schedules in order to obtain an outcome more to their liking. Neither committee member one, two, or three has any incentive to vote other than according to their strict schedule of preferences. Committee member four has a strong incentive to vote for $\mathrm{x}$ rather than $\mathrm{p}$. If we assume that he does, with four of five votes thus accounted for we can see that $p$ and $z$ will be eliminated on the first two ballots, and that in the final choice between $\mathrm{x}$ and $\mathrm{y}, \mathrm{x}$ will win. Thus, committee member four, going through this chain of reasoning, will conclude that he ought to vote for $x$, and $x$ will in fact be selected if all committee members act rationally. $X$ is of course the Condorcet choice, and our alternatives are single-peaked. Consider next a three-member committee with preferences yxqz, zyqx, and $\mathrm{xyqz}$, where $\mathrm{q}$ is no election (deadlock). If members adhere to their strict schedule of preferences the result is deadlock.Yet, as all practicing politicians know, a deadlock often has a habit of disappearing once it becomes clear that it exists. In this case because there does exist a candidate whom a majority prefer to deadlock, we would expect the deadlock never to materialize and y to be selected.

Theorem 4: For any set of preferences such that strict adherence to preference schedules by members yields a definite outcome, when the voting scheme is LCOR, given assumptions 1) - 7) above, if committee members preference schedules among all alternatives including the status quo $(q)$ are single-peaked, then the Condorcet choice (c) will be the outcome when committee members behave rationally.

Proof: We shall denote by $s$ that alternative which would be selected in LCOR were members to adhere strictly to their preference schedules. We shall show that rational behavior for members who prefer $c$ to $s$ (definition, a majority) is to vote for $c$ on the first ballot, thus insuring its selection. Clearly, if there are only two candidates this is what we mean by rational voting behavior. Our method of proof shall be to show by brute force that this procedure is rational for $n=3$, and then to extend the proof by induction. Consider the case where $n=3$. 
I. Let us assume that $c$ and $s$ coincide. If so, then the only rational reason $q$ committee member could have not to vote for $c$ would be in order to obtain the selection of a candidate whom he preferred to $c$. Assume, then, that there exist candidates $\mathrm{m}$ and $\mathrm{o}$ preferred by some members to $\mathrm{c}$. Assume further that sufficient committee members hold these preferences for $c$ to be eliminated on the first ballot. 21 The contest will then be between o and $\mathrm{m}$. Assume $\mathrm{m}$ wins. Given our assumption of perfect information this result is determinable in advance. The only members who will be pleased with this outcome will be those who prefer $\mathrm{m}$ to $\mathrm{c}$. Those who prefer $m$ to $c$ will be pleased at this outcome only if they also prefer $m$ to $c$. But those who prefer $m$ and o to $c$ are already counted among those who prefer $m$ co $c$. But those who prefer $m$ to $c$ must be in a minority of members, each of whom goes through the above chain of reasoning, will have any rational reason to vote for any candidate other than $c$ on the first ballot, and thus $c$ will be selected, contradicting our assumption that $c$ was eliminated on the first ballot. Moreover $c$ could defeat either $m$ or $o$ on the 2 nd ballot o by definition of the Condorcet choice.

II. Let us assume that $c$ and $s$ are distinct. Consider a committee member who favors $c$ to $s$. The only reason such a committee member could have not to vote for $c$ would be in order to obtain the selection of an alternative preferred by him to both $\mathrm{c}$ and $\mathrm{s}$. Denote this candidate $\mathrm{p}$. Assume further that the votes of those who prefer either $p$ or $s$ or both to $c$ are sufficient to ensure c's elimination on the first ballot. (Again, this assumption will lead to a contradiction.) The contest will then be between $s$ and $p$; but, by definition, $s$ will be selected. Hence, those committee members who prefer $c$ to $s$ (i.e., a majority of the committee) must, if they are rational, vote for $c$ on the first ballot if they are to forestall the selection of $s$, contradicting our assumption that $c$ was eliminated on the first ballot, and $A u$ Contraire leading to the conclusion that $c$ will be selected on the first ballot.

Consider now the case where $n=4$. If $c$ is not eliminated on the first ballot, we are done; for, once one candidate has been eliminated on the first ballot, we are back in the three candidate case, which we have already shown leads to the selection of c. Assume, then, that $c$ is eliminated on the first ballot. Consider preference schedules among the remaining three alternatives. Black has shown that any subset of a single-peaked set of preferences will itself be : single-peaked. Thus, among the three alternatives there will be some new Condorcet choice which we may denote $c^{\prime}$. In order to forestall the selection of $c^{\prime}$, and insure that of $c$, this majority must vote for $\mathrm{c}$ on the first ballot, contradicting our assumption that $\mathrm{c}$ was eliminated on the first ballot, and thus insuring that $c$ will be selected.

In like manner we may demonstrate that the Condorcet choice will be selectedfor $n=5,6, \ldots$ q.e.d.

Our second scheme, filling in the blanks (FIB), is also open to strategic manipulation through deviation from strict schedule of preferences. I leave the reader to devise his own examples. 
I have succeeded in proving a theorem for FIB analogous to theorem 4 for LCOR for all but one elusively refractory subcase. Since this proof is both long and incomplete I do not, however, bother to reproduce it here.

The third scheme we shall consider, SAP, is also open to manipulation. Consider a three member committee with preferences yxpzq, zypxq, xpzyq. If the order of alternatives is $x$ and $p$, then $z$, then $y$, and if members adhere to their strict schedule of preferences, then $y$ will be selected. Committee member 3 , however has an incentive to deviate from his schedule of preferences in a grand way. Assuming other members continue to adhere to their preference schedule, member three by voting for $\mathrm{p}$ rather than $\mathrm{x}$ and then for $\mathrm{z}$ rather than $\mathrm{p}$, may obtain the selection of $z$ rather than $y$. Indeed, anyone who attempted to ascertain three's real policy preferences from his voting behavior would be rather severly misled. While I suspect that a result similar to Theorem 4 may also hold for SAP, I have not yet succeeded in proving this.

\section{c)MANIPULATION OF THE ORDER IN WHICH ALTERNATIVES ARE VOTED UPON}

Of the schemes we have considered in this paper only SAP, of course, is subject to this type of manipulation. As our example shall show manipulation of the order of preferences may be of considerable importance under SAP. Thus, the preferences of the chairman and the rulcs for recognition may be crucial, particularly in the absence of either a Condorcet choice or perfect information. Consider a three-member committee with preference schedules xyqz, yzqx, and zxqy, respectively. There is no Condorcet choice since $x(p) y, z(p) x$, and $y(p) z$ with all alternatives being preferred to the status quo. Under SAP and assuming strict adherence to preference schedules, if the order is $x$ and $z$ then $y$, then $y$ will be chosen; if the order is $y$ and $z$ then $x$, then $x$ will be chosen; and if the order is $x$ and $y$ then $z$, then $z$ will be chosen. Common sense and the above example make clear the logic of the following theorem due to Black.

Theorem 5: Under SAP, given adherence to preference schedule, the later an alternative is entered into the balloting the better chance it will have to be selected. 2

Unfortunately, this theorem fails to hold if members are free to deviate from their strict schedule of preferences.

Let us consider the above example once again, this time with our committee members freed from the need to adhere strictly to their preferences schedules. If the order of alternatives is $x$ and $y$ then $z$, member one should vote for $y$ rather than $x$ on the first ballot, since he prefers $y$ to $z$. If he does not, then $z$ will be elected, since $z(p) x$. No other committee members have any incentive to switch votes on the first ballot since committee member three, who prefers $z$ to $y$, is 
already voting for $\mathrm{x}$; and committee member two, who prefers $\mathrm{y}$ to $\mathrm{z}$, is already voting for $y$. Similarly, if the order of balloting is $x$ and $x$ then $y$, committee member three, who prefers $x$ to $y$, should vote for $x$ rather than $z$ on the first ballot. Again, no other member has any incentive to switch votes. Committee member one prefers $x$ to $y$ and committee member two, who does not, is already voting for $\mathrm{x}$ on the first ballot. Finally, if the order of balloting is $\mathrm{y}$ and $\mathrm{z}$ then $\mathrm{x}$, committee member two should vote for $z$ rather than $y$ on the first ballot. Once again, no other committee members have any incentive to change their vote on either ballot. Thus, we obtain results rather different from those we obtained when we assumed strict adherence to schedules of preferences. In the example above, coming last in the balloting is no longer an advantage. In fact, quite the contrary.

\section{Conclusions}

The aim of this paper was to present elements of a rational theory of voting behavior, given various simplifying assumptions. For the case of single-peaked preferences we have made at least a start in that direction. We as yet, however, have far more cases than theorems to cover them. I hope, however, to have clearly demonstrated the potential importance of strategic deviations from strict preference schedules; and the range of strategic manipulations open to committee members to influence outcomes in their favor under various of the voting schemes in common use. Recognition of the conditions under which strategically motivated deviated from strict preference schedule is likely to occur may enable the observer to understand otherwise inexplicable deviations between observed voting behavior and avowed preferences or previously assigned scale position, or divergences in voting behavior by members with similar political beliefs in bodies of different size or political composition. Mathematical models, even if not fully descriptive of reality, amy, nonetheless serve valuable heuristic purposes. Theories of rational voting behavior may provide a standard to which the real world may be compared. A full theory of parliamentary strategy, however, must take into account not only deviations from the simplifying assumptions made herein, but also the modifications to standard parliamentary practices in use in a given committee or legislature (e.g. the cloture rule in the Senate, special recognition rules in Congress, amendment limitations established by the Rules Committee, etc.)

In the long run we wish a descriptive theory of political behavior; normative theory may provide a useful (and perhaps even invaluable) first step in the direction of that long-term goal. Normative theory, however, is of use only to the extent that it calls our attention to significant aspects of reality perhaps, even, by its very failure to take them into account.

\section{FOOTNOTES}

1 A voting scheme is less general than a social welfare function in that it need not be an ordering among all alternatives, but merely a partition of the set of alternatives into two classes: those which the committee approves and those which the committee does not. (A social welfare 
function is a process or rule, which, for each set of individual preference orderings states a corresponding social ordering of alternative social states. $[1]$, definition 4, p. 23.). A voting scheme is more general than a group decision function as defined by May [12] Murakami [16], and others in that it is not restricted to choices among two alternatives. Moreover, unlike a group decision function whose outcome always depends solely upon committee members preferences as determined by some omniscient observer, the outcome of a voting scheme is based upon preferences as expressed in individual voting decisions. To state the often neglected obvious, preferences as "revealed" in voting and "true" preferences need not coincide.

2Thus we cannot do justice to what William Riker [17] considers the heart of voting behavior; ammendments offered as a means of marshalling or demarshalling support for a given bill in which the amendments need not be and usually are not mutually exclusive. in a subsequent paper I hope to examine this important phenomenon in some detail.

${ }^{3}$ By a majority game we simply mean one in which if a majority of committee members decide to secure a certain outcome they can always do so.

${ }^{4}$ The relation $S$ is said to be a strong ordering relation if (a) for all $x$, not $x(S) x$; (b) for all $x, z, y$ either $x(S) y$ or $y(S) x ;(c)$ for all $x, y, z ;$ (S) $y$ and $y(S) z$ : imply $x(S) z-i, e$, if the relation is irreflexive, connected, and transitive.

It is important to note that a strong ordering of individual preferences does not guarantee either transitivity or the absence of ties in the preference ordering of the group. If a committee member prefers $x$ to $y$ to $z$, let us denote his preference schedule $x y z$. We denote the outcome of a voting scheme such that $x$ is preferred to $y x(p) y$. Now, consider a committee of two members whose preference schedules are $x y z$ and $y x z$ respectively. Individual preferences are strongly ordered, group preferences under the simple majority voting rule (cf. May [12]) are not since neither $x(p) y$ nor $y(p) x$. Similariy, consider a committee whose members have preference schedules $x y z, y z x$, and $z x y$ respectively. Under the simple majority voting rule, these preferences give rise to the famous voting paradox of cyclical majorities (Cf. Arrow [1], p. 3). Individual preferences are transitive, yet group preferences among paired afternatives are not, since $x(p) y$ and $y(p) z$, yet $z(p) x$. We may make this example even more explicit (Cf. Dah) [5], p. 42). Consider a committee of 101 members in which 1 member has preference ordering $x y z$, while fifty members have preferences $y z x$ and $z x y$, respectively. Fifty - one prefer $x$ to $y$; fifty - one prefer $y$ to $z$. If we assumed transitivity in collective choice it would follow that a majority of at least fifty - one preferred $x$ to $z$ also; but in fact only one member prefers $x$ to $z$, and the remaining one hundred committee members hold opposite preferences.

On the other hand, we may note that transitivity of group preferences does not imply transitivity of individual preferences. Consider a committee of three members. Member one prefers $x$ to $y$ and $y$ to $z$ and $z$ to $x$. Member two prefers $y$ to $x$ and $z$ to $y$ and $x$ to $z$. Member three prefers $x$ to $y$ and $y$ to $z$ and $x$ to $z$. Under simple majority voting the group's preferences are transitive since $x(p) y, y(p) z$ and $x(p) z$, but the preference schedules of members one and two are intransitive. (Cf. David C. McGarvey, "A Theorem on the Construction of Voting Paradoxes," Econometrica, 1953, pp. 608-610.)

5 We may argue that this assumption is not as unrealistic as it might first seem. In small committee members soon learn a great deal about each other's true preferences, and in larger legislative bodies knowledge of past voting behavior and of constraints such as party affiliation or constituency composition may make preferences readily predictable. (See, for example, Duncan MacRae, DIMENSIONS OF CONGRESSIONAL VOTING, U. of California Press, 1958).

6 By perfect information is meant knowledge of where one is on the game tree being available to all players at all times. (A discussion of game trees, information sets, etc. may be found in Luce and Raiffa, GAMES AND DECISIONS, New York, 1957, pp. 39-56.). In general, only roll - call voting procedures enable committee members to possess perfect information. In 
order to satisfy our assumption of perfect information for voting schemes requiring written ballots we shall make the additional assumption that (labelled) ballots of all committee members are avallable for inspection after each round of balloting.

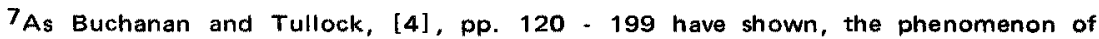
logrolling, in which different issues and different votes are interconnected, enables us to understand how different intensities of preferences may be reconciled in a one - man one vote context. Thus, our work is open to the same criticism which Tullock levels against Black when he asserts (IBID, p. 328) that "the work of a voting scheme can be analyzed only as it produces results over a series of issues." I consider this criticism to be an important one to which my only defense can be that my wark, and that of Black upon which it is largely based, are both intended as prologemena to a more comprehensive theory of voting behavior which takes this criticism into account.

8 We assume that utility is gained by the selection of candidate or candidates of one's choice. We neglect the possibility of utility being gained merley from being on the winning side or merely from voting for a given candidate regardless of his chance of election. Thus, we neglect future minded voting behavior (e.g., voting for a given candidate in order to enhance his chances of winning some future election or failing to vote for a candidate to motivate him to change his policies in the future). We shall assume explicitly that each election in entire unto itself. We also assume that the margin of success of the winning candidate (s) is irrelevant to committee members.

9Named for the Marquis de Condorcet (1743-1794), who was apparently the first to propose it. Is/e/e Black [2] pp. $159-172$. It is clear that a Condorcet choice need not always exist. When it does exist, it is, given our assumption of strong preference orderings, unique.

Black [2], pp. 25 - 32 has shown the important result that when preference scheduies are single - peaked (see below) a Condorcet choice always exists. This condition, it is readily appparent, is sufficient but not necessary. We note that the existence of a Condorcet choice is equivalent to the existence of a "top," ( $[8]$, p. 36$)$ i.e., that it is not the case that for every outcome there is another outcome which a majority of voters would have preferred. In $[8]$ are given other sufficient but not necessary conditions for the existence of a Condorcet choice. Neither they nor I, however, have found conditions which are both necessary and sufficient.

Since the removal of an alternative would not affect the single - peakedness, each subset of a single-peaked set of alternatives will contain an alternative satisfying the Condorcet property for alternatives in the subset. Furthermore, the alternative which satisfies the Condorcet criterion in a given subset will satisfy the criterion in any smaller subset (although, of course, not necessarily in any larger one).

We shall assume that a Condorcet choice, if it exists, is always available as one of the alternatives. In fact, as Tullock has noted in [11], p. 38, if alternatives are discontinuous (e.g., at least $\$ 5.00$ apart) then the Condorcet choice may not be avallable as an alternative. (1t might, say, be \$102.63). We shall, however, neglect this possibility.

By a committee member's adherence to his strict schedule of preferences we shall mean his always voting for that alternative of those not yet eliminated which stands highest on his preference schedule. In filling in the blanks, adherence of strict schedules of preferences requires that members not vote in favor of any alternative until all alternatives preferred to it by them have been eliminated from consideration.

10 Arrow [1], p. 77, gives a more formał definition in terms of preference orderings. To require that all preference schedules be single-peaked with respect to some possible ordering of alternatives is to posit an "underlying one-dimensionat variable." (IBID, p. 75.) Single-peaked 
preferences have a number of nice properties (e.g., Arrow [1], pp. 78-80, the "Possibility Theorem for Single Peaked Preferences,"), and we shall cite various theorems of Black [2] regarding them, as well as proving several interesting theorems of our own.

A number of political scientists have studied legislative voting behavior and have discovered significant issue continua which satisfy the single-peakedness restriction. See Duncan MacRae, DIMENSIONS OF CONGRESSIONAL VOTING, U. of California Press.

Dummett and Farguhavson [8] show that when preference schedules are single peaked then there exists a stable outcome. (A situation is called stable if no group of committee members by voting differently (those outside the group voting the same as before) could have obtained a result which the members of the group would have preferred. An outcome is said to be stable if it contains some stable situation.) It is obvious that not every situation in a stable outcome could be stable except in the trivial case of only one alternative. In general, a stable outcome may contain more than one stable situation. The definition of stability above is a property solely of the committee members'preference orderings. For various voting schemes in the case in which preferences are single-peaked we shall show that stable situations are those actually attained when committee members behave rationally.

11 In the usual symbolic logic notation let $A$ be the proposition that the voting scheme always yields the Condorcet choice as outcome when members adhere to their strict schedule of preferences. Let $B$ be the proposition that a voting scheme satisfies the criterion of independence of irrelevant alternatives (CIIA) for the set of preferences schedules under consideration. Let $\mathbf{C}$ be the proposition that there exists a Condorcet choice for the set of preference schedules under consideration. Let $D$ be the proposition that in the two alternative case the voting scheme reduces to the method of simple majority decision. Let $E$ be the proposition that the voting scheme always yield a definite outcome when members adhere to their strict schedules of preferences. Theorem $\mid$ asserts that $(E \wedge C \wedge A) \rightarrow B$. Theorem 2 asserts that $(E \cap D \wedge B) \rightarrow A$, from which follows the collary $(D \wedge B) \rightarrow(A \sim E)$. Since we know that $A \rightarrow E$ and $A \rightarrow C$ we easily obtain $A \rightarrow B$, hence $\left(E_{\wedge} D \wedge\right) \rightarrow B$ hence given $E A D, A \leftrightarrow B$.

It is possible for there to exist a voting scheme and a set of preference schedules in which no Condorcet choice exists such that members' adherence to their strict schedule of preferences still yields a definite outcome and the scheme still satisfies CIIA for the given set of preferences. To see this, merely consider tyranny. Similarly, it is possible for a scheme to satisfy CIIA but not necessarily to yield a definite outcome. Consider the unanimity scheme.

12 Interestingly enough, this scheme, despite its common use, is not mentioned in ROBERT'S RULES OF ORDER [19], which prescribes for elections the same procedure as for amendments (described below under the heading SAP) save that there is no final vote against the status quo.

We shall use the term candidates whenever the voting scheme we are considering is more commonly used for elections than for choice among proposals.

13 Black [2], p. 76. Of course Black (and we) use the term extremist nonpejoratively. Extremists refer to candidates highly ranked by some committee members while ranked very low by most of the others; moderates will refer to candidates neither strongly favored nor strongly opposed by most committee members.

14This procedure is that labelled Procedure[2], pp. 21, and is referred to as the standard amendment procedure because it is just that in American parliamentary procedure Is/e/e Robert, [19], pp. 134-135.) Minus the final vote against the status quo it is also the procedure recommended by Robert for elections. In this form it may give rise to the selection of a candidate not preferred to the status quo of no election at all. IBID, pp. 148-52, does mention an alternative procedure for dealing with amendments which are clearly unidimensionally scalable (e.g. pertaining to time, money, distance, etc.) This method, known as "filling in the blanks," we take up below. 


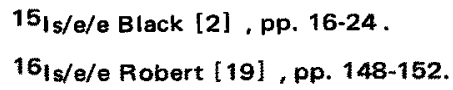

17 In another paper I have made explicit the equivalence between unidimensional (Guttman) scalability and single-peakedness.

Robert [19] takes for granted that the first outcome which attains a majority will be preferred by a majority over all other outcomes, l.e. will be the Condorcet choice. However, If committee members vote for any alternative which they favor to the status quo, then the first alternative to command a majority will be selected and that alternative need not be the Condorcet choice. Consider a committee in which all alternatives proposed are preferred by a majority to the status quo. Unless the Condorcet choice is the first alternative voted upon it will never be considered.

18 Cited in Black [2], p. 182.

19 In fairness we must note that a number of authors including Arrow [1], Black [2]; Dahl [5], Downs [6], and Riker [17] cite instances in which strategically minded deviations from strict preference schedules might be expected or actually took place. But, in general, the analysis of these situations was confined to what Majumdar [11], fn. 5, p. 72, calls "first order" camouflages.

As Dummett and Farquharson [8], p. 34, note "The only hypothesis which would make the assumption of uniformly sincere voting plausible would be the absurdly restrictive one that no voter had any knowledge, before or during the voting, of the preference scales of others." Other than studies of $n$ person games in which cardinal utilities are introduced, the only author I'm aware of who has attempted to analyse strategically motivated deviations from preference schedules is Tapas Majumdar [11], in a brief article offering a rather unsatisfactory treatment of a particular majority voting game in which the order in which alternatives are posed is of decisive importance.

20 Downs [7], pp. 48-49.

21 We neglect the possibility of deadlock since a majority prefer $c$ to $q$.

22 Black [2], p. 40 .

\section{REFERENCES}

[1] Arrow, Kenneth, Social Choice and Individual Values, 2nd Edition, Wiley, New York, 1951.

[2] Black, Duncan, Theory of Committees and Elections, The, Cambridge University Press, 1958.

[3] Blau, Julian H., "The Existence of Social Welfare Functions," Econometrica, Vol. XXV, April, 1957, pp. 302-313.

[4] Buchanan, James M., and Tullock, Gordon, Calculus of Consent, The, University of Michigan Press, Ann Arbor, 1962.

[5] Dahl, Robert A., Preface to Democratic Theory, A, University of Chicago Press, 1956. 
[6] De Grazia, Sebastian, "Mathematical Derivation of an Election System," ISIS, Vol. XLIV, June, 1953.

[7] Downs, Anthony, Economic Theory of Democracy, An, Harper and Row, New York, 1957.

[8] Dummett, Michael and Farguharson, Robin, "Stability in voting," Econometrica, Vol. XXIX, January, 1961, pp. 33-43.

[9] Duverger, Maurice, Political Parties, 2nd Edition, Wiley, New York, 1959, pp. 206-372.

[10] Inada, Ken-Ichi, "Alternative Incompatible Conditions for a Social Welfare Function," Econometrica, vol. XXIII, 1955, pp. 396-399.

[11] Majumdar, T., "Choice and Revealed Preference," Econometrica, vol. 24, 1956, pp. 71-73.

[12] May, Kenneth O., "A Set of Necessary and Sufficient Conditions for Simple Majority Decisions," Econometrica, Vol. 21, 1953, pp. 172-173.

[13] _ "A Note on the Complete Indpendence of the Conditions for Simple Majority Decision," Econometrica, Vol. 21, 1953, pp. 172-173.

[14] _ "Intransitivity, Utility, and the Aggregation of Preference Patterns," Econometrica, Vol. 29, April, 1961, pp. 244-246.

[15] Murakami, Yasusuke, "A Note on the General Possibility Theorem of the Social Welfare Function," Econometrica, vol. 29, April, 1961, pp. 244-246.

Institute for Research in the Behavioral Economic and Management Sciences Paper No. 90, Purdue University, 1964.

[17] Riker, William, Democracy in the United States, 2nd Edition, Macmillan, 1965, pp. 150-186.

[18] "Voting and the Summation of Preferences," American Political Science Review, 1962, pp. 900-911.

[19] Robert, Henry M., Robert's Rules of Order Revised, 75th Anniversary Edition, Scott Foresman, 1951. 
[20] Tullock, Gordon, "The General Irrelevance of the General Impossibility Theorem," Quarterly Joumal of Economics, May, 1967.

[21] Michigan Press, Ann Arbor, 1967, esp. pp. 1-50. 